$\underset{\text { clinical }}{\text { nephron }}$

Practice
Nephron 2016;134:20-24

DOI: 10.1159/000445844
Received: December 23, 2015

Accepted after revision: March 26, 2016

Published online: April 21, 2016

\title{
Progression, Remission and Regression of Chronic Renal Diseases
}

\author{
Monica Cortinovis ${ }^{\mathrm{a}}$ Piero Ruggenenti ${ }^{\mathrm{a}} \mathrm{b}$ Giuseppe Remuzzi ${ }^{\mathrm{a}-\mathrm{c}}$ \\ aIRCCS - Istituto di Ricerche Farmacologiche Mario Negri, Clinical Research Center for Rare Diseases 'Aldo and Cele \\ Daccò', and 'bunit of Nephrology and Dialysis, Azienda Ospedaliera Papa Giovanni XXIII, Bergamo, and 'Department \\ of Biomedical and Clinical Sciences, University of Milan, Milan, Italy
}

\section{Key Words}

Chronic renal disease $\cdot$ Remission $\cdot$ Regression .

Renin-angiotensin system

\begin{abstract}
Progression to end-stage renal disease is common in chronic nephropathies, independent of the initial insult. While genetic factors may contribute to susceptibility and progression of renal disease, proteinuria has been documented as an independent predictor of outcome. Reduction of urinary protein levels by restoration of glomerular sieving function with renin-angiotensin system (RAS) blockers has been shown to limit renal function decline in individuals with nondiabetic and diabetic nephropathies to the point that remission of the disease and regression of renal lesions have been observed in experimental animals and even in humans. In animal models, regression of glomerular structural changes is associated with remodeling of glomerular architecture. This review briefly describes our understanding of the mechanism of renal disease progression, the therapeutic advantages of ameliorating glomerular sieving dysfunction and proteinuria of RAS inhibitors and how remission/regression of renal injury can be achieved with multifactorial interventions.

(c) 2016 S. Karger AG, Basel
\end{abstract}

Chronic kidney disease (CKD) is a key determinant of poor health outcome for major non-communicable diseases that are the leading cause of death in the world. It has been estimated that diseases of the kidney account for more than 950,000 deaths every year, and the number of patients with end-stage renal disease (ESRD) now receiving renal replacement therapy (RRT) worldwide exceeds 1.4 million. Thus, efforts to prevent or halt renal disease progression are of utmost importance to reduce the clinical and economic consequences of CKD.

\section{Mechanisms of Renal Disease Progression}

In the past 3 decades, research in animals and humans has helped our understanding of the mechanism of CKD progression. These studies have established that most renal diseases progress to renal failure as a consequence of functional adaptations intervening in the kidney, after the original disease process causes an initial loss of nephron

Contribution from the Special Symposium to celebrate the contributions of David G. Warnock, MD to Academic Nephrology, The University of Alabama at Birmingham, Division of Nephrology, November 9, 2015.

\section{KARGER}

() 2016 S. Karger AG, Basel

E-Mail karger@karger.com

www.karger.com/nef
Prof. Giuseppe Remuzzi

IRCCS - Istituto di Ricerche Farmacologiche 'Mario Negri'

Clinical Research Center for Rare Diseases 'Aldo and Cele Daccò'

Via GB Camozzi 3, Ranica, IT-24020 Bergamo (Italy)

E-Mail giuseppe.remuzzi@ marionegri.it 
Table 1. Definitions of progression, remission and regression of proteinuric chronic nephropathy

\begin{tabular}{llll}
\hline Variable & Progression & Remission & Regression \\
\hline Proteinuria & $\geq 1 \mathrm{~g} / 24 \mathrm{~h}$ & $<1 \mathrm{~g} / 24 \mathrm{~h}$ & $<0.3 \mathrm{~g} / 24 \mathrm{~h}$ \\
GFR & Declining* & Stable & Increasing \\
Renal structural changes & Worsening & Stable & Improving \\
\hline
\end{tabular}

* Faster than physiological decline associated with aging $\left(0.1 \mathrm{ml} / \mathrm{min} / 1.73 \mathrm{~m}^{2}\right.$ per month).

units [1]. Such changes, extensively studied in rodents, include glomerular hyperperfusion and hypertension of the remaining nephrons, which begin with enhancing the filtration capacity of single nephrons. These changes initially minimize the functional consequences of nephron loss but ultimately become detrimental, contributing to the impairment of the glomerular barrier's size selective properties and inducing protein ultrafiltration. An excess of protein content in Bowman's space and in the lumen of tubules eventually results in the activation of inflammatory and apoptotic pathways, fuelling progressive renal damage [1]. Indeed, an excessive protein load of podocytes can be a factor underlying progressive injury of these glomerular cells that, by releasing transforming growth factor- $\beta 1$ in the microenvironment, ultimately allow myofibroblast differentiation of mesangial cells [1]. Moreover, excessive protein reabsorption by proximal tubuli provides further intrinsic toxicity to this nephron segment. Thus, both in-vitro and in-vivo protein overload causes an increased production of vasoactive and inflammatory mediators such as endothelin-1, monocyte chemoattractant protein-1, RANTES and interleukin-8 [1]. The activation of a variety of cytokines, growth factors and vasoactive substances may result in abnormal accumulation in the interstitium of extracellular matrix collagen, fibronectin and other components that are responsible for interstitial fibrosis [1]. Pro-inflammatory mediators promote local recruitment of macrophages and lymphocytes that, in turn, can stimulate the transformation of interstitial cells into myofibroblasts. Renoprotection is a strategy that aims to interrupt or reverse this process of progressive renal injury (table 1).

\section{Renoprotection by RAS Blockade in Proteinuric Non- Diabetic Nephropathies}

Current strategies to prevent or revert renal disease progression focus on reduction of protein trafficking along with strict blood pressure control. Blockade of the renin-angiotensin system (RAS) by means of angiotensin-converting enzyme inhibitors (ACEi) and/or angiotensin II type 1 receptor blockers (ARBs) is the most effective treatment to achieve these purposes. Animal models of chronic nephropathy have clearly documented that RAS inhibitors reduce intraglomerular hydraulic pressure and improve the selectivity of the glomerular barrier, an effect that translates into a reduction of proteinuria and prevention of glomerulosclerosis [2]. Similar effects have been reported on the clinical ground. In individuals with non-diabetic chronic nephropathies the ramipril efficacy in nephropathy (REIN) trial showed that, at equivalent blood pressure control, the ACEi ramipril halved the rate of measured glomerular filtration rate (GFR) decline and progression to ESRD compared to placebo. Notably, ramipril-treated patients also enjoyed a great decrease in proteinuria that inversely correlated with the rate of GFR decline, providing evidence for a nephroprotective effect of protein trafficking reduction [3]. Renoprotection was time dependent, and in patients on continued ramipril therapy for at least 5 years, the rate of GFR decline progressively improved up to $1 \mathrm{ml} / \mathrm{min} / 1.73 \mathrm{~m}^{2}$ per year, approximating the physiological age-related loss of GFR with time in individuals with no evidence of renal disease (fig. 1) [4]. Post-hoc analysis of the REIN study also showed that ACE inhibition therapy should be offered to all patients with proteinuric chronic nephropathies, regardless of renal function [5]. Maximal renoprotection was achieved when the treatment started early in the course of the disease (GFR $>50 \mathrm{ml} / \mathrm{min} / 1.73 \mathrm{~m}^{2}$ ). However, treatment is also renoprotective for levels of renal function between 10 and $30 \mathrm{ml} / \mathrm{min} / 1.73 \mathrm{~m}^{2}$, indicating the need not to withhold ACEi, even when GFR approximates to levels requiring RRT [5].

Combination therapy with ACEi and ARB inhibits the RAS more efficiently than does each agent alone through an additive effect. Consistently, the results of a meta-analysis indicated that dual RAS blockade was associated with greater proteinuria reduction compared to ACEi or ARB monotherapy in patients with chronic nephropathy [6]. 


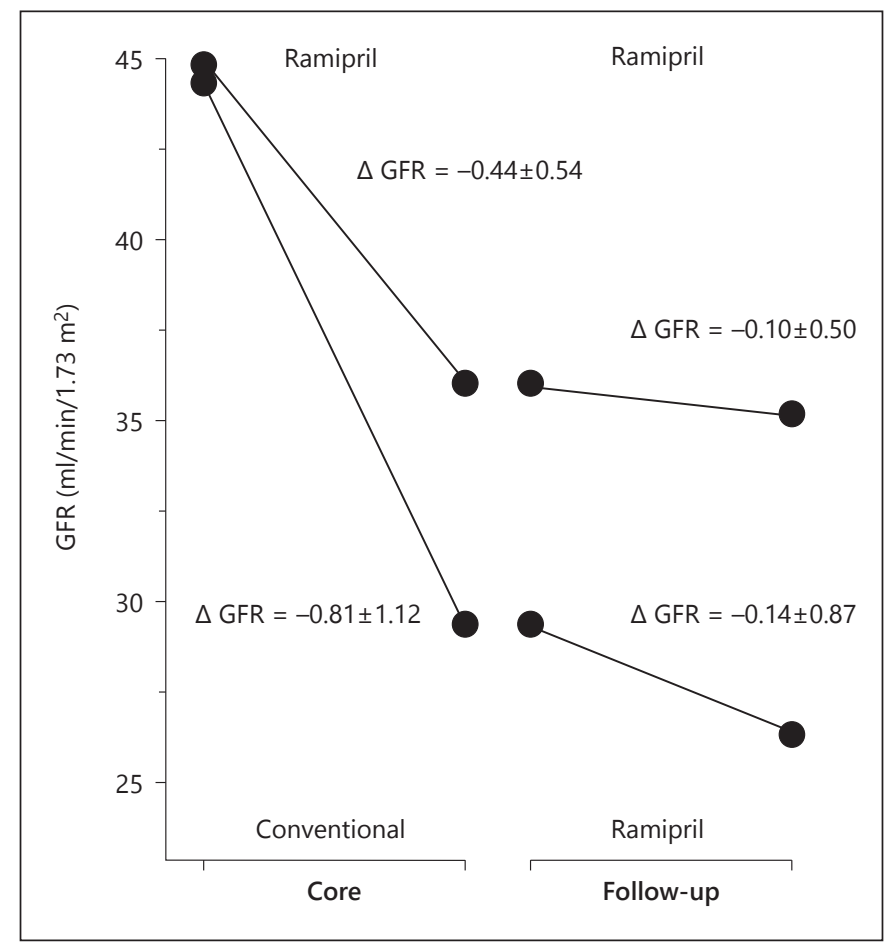

Fig. 1. Mean GFR decline during the REIN core and follow-up study in patients continued on or switched to ramipril. Mean of the differences of GFR decline between the core and the follow-up study was 0.34 (95\% CI 0.08-0.6) and 0.66 (95\% CI 0.17-1.15) $\mathrm{ml} /$ $\mathrm{min} / 1.73 \mathrm{~m}^{2}$ per month in patients continued on or switched to ramipril, respectively.

Concerns have been raised about double RAS inhibition since combined treatment of ramipril and telmisartan increased the risk of the pre-specified composite end point of any dialysis, serum creatinine doubling or death compared with either drug alone in a large study comprising 25,620 patients with atherosclerotic disease and/or diabetes with end-organ damage [7]. However, the excess of adverse renal outcomes on combination therapy was largely driven by the more frequent need for short-term dialysis, conceivably a treatment-related acute effect on renal hemodynamics that is reversible upon treatment withdrawal, rather than an indication of CKD progression. Moreover, $96 \%$ of patients in this study had normoor micro-albuminuria, pointing to limited kidney damage to halt or reverse. Debate about the role of dual agent RAS blockade in the management of CKD has recently been reignited by the results of a meta-analysis showing that ACEi and ARB combination therapy is the most effective approach to prevent ESRD in patients with diabetic kidney disease [8]. This treatment benefit was independent of blood pressure control, but was associated with great improvement in albuminuria [8]. The above findings can be taken to suggest that dual RAS inhibition could be a powerful tool to slow or prevent the progression of chronic proteinuric nephropathies, provided that treatment is accompanied by close monitoring of renal function and serum electrolytes, adjusting drug doses accordingly.

\section{Remission, Regression and Kidney Repair}

Remission and regression of renal lesions could have a significant impact on progressive renal disease and its sequelae. Evidence that regression of CKD and the underlying lesion is achievable in humans can only be indirect, but it is fairly consistent and encouraging [9]. In 10 of REIN's study patients on ACEi therapy, there was a break point indicating the transition from an initial phase of progressive GFR decline to a second phase of gradual renal function improvement, possibly reflecting regression of renal lesions along with some degree of kidney regeneration. The beneficial effect of ramipril on GFR was paralleled by further proteinuria reduction across the break point [10]. Subsequent experimental studies showed that amelioration of renal function achieved by chronic ACEi therapy was mediated through regression of sclerosis in most partially injured glomeruli and regeneration of kidney vasculature. Instrumental to this discovery were 3-dimensional reconstruction studies of the glomerular capillary tuft, which allowed an accurate quantification of sclerosis volume reduction upon treatment $[11,12]$.

Regression of glomerulosclerosis and neoformation of glomerular tissue have been linked to progenitor/stem cells of renal or extrarenal origin. A key observation was that human renal cell progenitors isolated from the urinary pole of the Bowman's capsule could differentiate into podocytes and tubular cells in vitro, and induced tubular regeneration and amelioration of kidney structure when infused into immunodeficient mice with acute kidney injury [13]. In rats with extracapillary hyperplastic lesions and in patients with extensive podocyte loss, ACEi therapy sustained glomerular repair by modulating progenitor cell proliferation, limiting crescents generation and promoting podocyte repair $[14,15]$.

Moreover, experimental studies have shown that along with ACEi, prednisone, Notch inhibitors, blockers of chemokine stromal cell-derived factor- 1 and retinoids can be added to the list of agents that improve podocyte regeneration by augmenting the number of parietal epithelial cell progenitors [16]. 


\section{Remission/Regression with Multifactorial Intervention}

A significant cohort of patients treated with RAS blockers, however, shows only partial anti-proteinuric response, eventually heralding a progressive loss of renal function in most cases. To overcome this shortcoming, the Unit of Nephrology, Bergamo Hospital, Bergamo, Italy, has designed a multimodal intervention protocol - the Remission Clinic program - targeting urinary proteins by dual RAS inhibition with ACEi and ARBs up-titrated to maximum tolerated doses, by intensified blood pressure control, by amelioration of dyslipidemia with statins, smoking cessation, lowering salt intake and healthy lifestyle implementation in patients who had overt proteinuria despite ACEi therapy $[17,18]$. During a 7 -year follow-up, only 2 of 56 patients treated according to the Remission Clinic strategy progressed to ESRD compared to up $50 \%$ of matched historical reference patients who had received ACEi therapy titrated to target blood pressure [17]. Results of this proof-of-concept observational study have been currently confirmed in a large outpatient clinical cohort of CKD patients with overt nephropathy, demonstrating that this approach can be safely and effectively applied in everyday practice. Preliminary results in more than 1,000 CKD patients with mean proteinuria of $3.0 \pm$ $2.6 \mathrm{~g} /$ day, mean estimated GFR of $60.7 \pm 30.7 \mathrm{ml} / \mathrm{min} /$ $1.73 \mathrm{~m}^{2}$ at baseline and more than 4-year follow-up showed that $60 \%$ of individuals had remission or regression of their renal disease progression (P. Ruggenenti, personal communication). The proposed therapy was safe. Together, the above observations indicate that this standardized intervention program can be safely and effectively used to normalize proteinuria and to slow the loss of renal function significantly, especially in patients without type 2 diabetes and with otherwise rapidly progressive chronic nephropathies. Indeed, the remission clinic has highlighted that in approximately two-thirds of type $2 \mathrm{di}-$ abetic patients with overt nephropathy, remission of proteinuria could not be achieved through the multimodal approach, and renal and cardiovascular risks remained elevated. These very patients urgently need novel therapeutic interventions that would complement the effects of RAS inhibitors in order to improve clinical outcomes.

These findings also point out the importance of early pharmacologic intervention in patients with type 2 diabetes. In a rat model of streptozotocin-induced diabetes, ACE inhibition afforded remarkable renoprotection against the development of nephropathy, but only when treatment was started early in the course of the disease

Progression, Remission and Regression of Chronic Renal Diseases
[19]. In the IRbesartan MicroAlbuminuria type 2 diabetes in hypertensive patients (IRMA-2) trial, full-dose irbesar$\tan$ therapy reduced the 2-year incidence of progression to macroalbuminuria from 14.9 to $5.2 \%$ compared to placebo and increased the rate of regression to normoalbuminuria [20]. RAS inhibition is renoprotective even when treatment is started earlier, as shown in the Bergamo Nephrologic Diabetes Complication Trial in type 2 diabetic patients with hypertension and normal urinary protein excretion rate [21]. Four years treatment with the ACEi trandolapril reduced the risk of progression to microalbuminuria from 10.9 to $5.8 \%$ compared to non-ACEi therapy [21]. Interestingly, among normoalbuminuric patients, any measurable albuminuria bore significant cardiovascular risk and in those receiving ACEi therapy, the event rate was uniformly low [22]. A recent study using a statistical model able to simulate diabetic kidney disease progression confirmed and extended previous findings. Indeed, RAS intervention in the early stages of the disease was more beneficial in delaying ESRD than later treatment [23].

\section{Conclusions}

Most chronic nephropathies progress relentlessly to ESRD, since they lack a specific treatment. Research in animals and patients has helped our understanding of the mechanisms of this progression and has indicated possible approaches for renoprotection. Slowing progression, promoting remission or even regression of chronic renal disease has been based on control of blood pressure and reduction of proteinuria with inhibitors/blockers of RAS. For those with non-diabetic overt nephropathies despite conventional RAS therapy, the multimodal protocol of the remission clinic may represent a suitable tool for management of renal disease progression. Experimental and clinical research should now focus more on diabetic kidney disease where treatment management of disease progression remains largely unsatisfactory, unless early stages are tackled. Greater knowledge regarding the pathogenic pathways of glomerulosclerosis/renal fibrosis and regression of kidney lesions, as well as a better understanding of how to influence these processes, will hopefully reveal new paths to reverse or at least halt the decline of renal function in patients with renal disease.

\section{Disclosure Statement}

The authors declare no conflicts of interest. 


\section{References}

$>1$ Zoja C, Abbate M, Remuzzi G: Progression of chronic kidney disease: insights from animal models. Curr Opin Nephrol Hypertens 2006; 15:250-257.

-2 Remuzzi G, Benigni A, Remuzzi A: Mechanisms of progression and regression of renal lesions of chronic nephropathies and diabetes. J Clin Invest 2006;116:288-296.

-3 Randomised placebo-controlled trial of effect of ramipril on decline in glomerular filtration rate and risk of terminal renal failure in proteinuric, non-diabetic nephropathy. The GISEN group (gruppo Italiano di studi epidemiologici in nefrologia). Lancet 1997;349: 1857-1863.

4 Ruggenenti P, Perna A, Gherardi G, et al: Renal function and requirement for dialysis in chronic nephropathy patients on long-term ramipril: REIN follow-up trial. gruppo Italiano di studi epidemiologici in nefrologia (GISEN). Ramipril efficacy in nephropathy. Lancet 1998;352:1252-1256.

$\checkmark 5$ Ruggenenti P, Perna A, Remuzzi G: ACE inhibitors to prevent end-stage renal disease: when to start and why possibly never to stop: a post hoc analysis of the REIN trial results. Ramipril efficacy in nephropathy. J Am Soc Nephrol 2001;12:2832-2837.

$\checkmark 6$ Catapano F, Chiodini P, De Nicola L, et al: Antiproteinuric response to dual blockade of the renin-angiotensin system in primary glomerulonephritis: meta-analysis and metaregression. Am J Kidney Dis 2008;52:475-485.

-7 Mann JF, Schmieder RE, McQueen M, et al: Renal outcomes with telmisartan, ramipril, or both, in people at high vascular risk (the ONTARGET study): a multicentre, randomised, double-blind, controlled trial. Lancet 2008;372:547-553.
-8 Palmer SC, Mavridis D, Navarese E, et al: Comparative efficacy and safety of blood pressure-lowering agents in adults with diabetes and kidney disease: a network metaanalysis. Lancet 2015;385:2047-2056.

9 Ruggenenti P, Schieppati A, Remuzzi G: Progression, remission, regression of chronic renal diseases. Lancet 2001;357:1601-1608.

10 Ruggenenti P, Perna A, Benini R, et al: In chronic nephropathies prolonged ACE inhibition can induce remission: dynamics of time-dependent changes in GFR. Investigators of the GISEN group. Gruppo Italiano studi epidemiologici in nefrologia. J Am Soc Nephrol 1999;10:997-1006.

11 Remuzzi A, Gagliardini E, Sangalli F, et al: ACE inhibition reduces glomerulosclerosis and regenerates glomerular tissue in a model of progressive renal disease. Kidney Int 2006; 69:1124-1130.

12 Remuzzi A, Sangalli F, Macconi D, et al: Regression of renal disease by angiotensin II antagonism is caused by regeneration of kidney vasculature. J Am Soc Nephrol 2016;27:699705.

13 Sagrinati C, Netti GS, Mazzinghi B, et al: Isolation and characterization of multipotent progenitor cells from the Bowman's capsule of adult human kidneys. J Am Soc Nephrol 2006;17:2443-2456.

14 Benigni A, Morigi M, Rizzo P, et al: Inhibiting angiotensin-converting enzyme promotes renal repair by limiting progenitor cell proliferation and restoring the glomerular architecture. Am J Pathol 2011;179:628-638.
15 Rizzo P, Perico N, Gagliardini E, et al: Nature and mediators of parietal epithelial cell activation in glomerulonephritides of human and rat. Am J Pathol 2013;183:1769-1778.

16 Romagnani P, Lasagni L, Remuzzi G: Renal progenitors: an evolutionary conserved strategy for kidney regeneration. Nat Rev Nephrol 2013;9:137-146.

17 Ruggenenti P, Perticucci E, Cravedi P, et al: Role of remission clinics in the longitudinal treatment of CKD. J Am Soc Nephrol 2008; 19:1213-1224.

18 Vegter S, Perna A, Postma MJ, et al: Sodium intake, ACE inhibition, and progression to ESRD. J Am Soc Nephrol 2012;23:165-173.

19 Perico N, Amuchastegui SC, Colosio V, et al: Evidence that an angiotensin-converting enzyme inhibitor has a different effect on glomerular injury according to the different phase of the disease at which the treatment is started. J Am Soc Nephrol 1994;5:1139-1146.

20 Parving $\mathrm{HH}$, Lehnert $\mathrm{H}$, Bröchner-Mortensen J, et al: The effect of irbesartan on the development of diabetic nephropathy in patients with type 2 diabetes. N Engl J Med 2001;345:870878.

21 Ruggenenti P, Fassi A, Ilieva AP, et al: Preventing microalbuminuria in type 2 diabetes. N Engl J Med 2004;351:1941-1951.

-22 Ruggenenti P, Porrini E, Motterlini N, et al: Measurable urinary albumin predicts cardiovascular risk among normoalbuminuric patients with type 2 diabetes. J Am Soc Nephrol 2012;23:1717-1724.

23 Schievink B, Kröpelin T, Mulder S, et al: Early renin-angiotensin system intervention is more beneficial than late intervention in delaying end-stage renal disease in patients with type 2 diabetes. Diabetes Obes Metab 2016;18:64-71. 